\title{
New integrable models and analytical solutions in $f(R)$ cosmology with an ideal gas
}

\author{
G. Papagiannopoulos, ${ }^{1, *}$ Spyros Basilakos, ${ }^{2, \dagger}$ John D. Barrow, ${ }^{3,}$ and Andronikos Paliathanasis ${ }^{4,5, \S}$ \\ ${ }^{1}$ Faculty of Physics, Department of Astronomy-Astrophysics-Mechanics University of Athens, \\ Panepistemiopolis, Athens 157 83, Greece \\ ${ }^{2}$ Academy of Athens, Research Center for Astronomy and Applied Mathematics, \\ Soranou Efesiou 4, 11527 Athens, Greece \\ ${ }^{3}$ DAMTP, Centre for Mathematical Sciences, University of Cambridge, \\ Wilberforce Road, Cambridge CB3 OWA, United Kingdom \\ ${ }^{4}$ Instituto de Ciencias Físicas y Matemáticas, Universidad Austral de Chile, Valdivia 5090000, Chile \\ ${ }^{5}$ Institute of Systems Science, Durban University of Technology, \\ PO Box 1334, Durban 4000, Republic of South Africa
}

(Received 31 October 2017; published 23 January 2018)

\begin{abstract}
In the context of $f(R)$ gravity with a spatially flat FLRW metric containing an ideal fluid, we use the method of invariant transformations to specify families of models which are integrable. We find three families of $f(R)$ theories for which new analytical solutions are given and closed-form solutions are provided.
\end{abstract}

DOI: $10.1103 /$ PhysRevD.97.024026

\section{INTRODUCTION}

The discovery of the accelerated expansion of the Universe has led to many cosmological models which aim to explain this phenomenon, using a spatially flat geometry and a cosmic dark sector formed by cold dark matter and some form of dark energy, with negative pressure. Among the large family of possible cosmological scenarios, the modified gravity models occupy a muchstudied position in cosmological studies, since they provide a way of explaining the accelerated expansion of the universe, under a modification of Einstein-Hilbert action. Traditionally, the simplest term which has been added to the gravitational action is the squared Ricci scalar which leads to a model of inflation [1,2]. The latter corresponds to a family of models in which the gravitational action is a function of the Ricci scalar of the underlying geometry and consequently the cosmological field equations are fourthorder in time. This class of models belongs to the so called $f(R)$ extended theory of gravity [3-5], and it has been applied in various areas of the gravitational and cosmological studies (see [6-12] and references therein).

The modification of the Einstein-Hilbert action by introducing other kind of invariants has lead to an entire menagerieof $f$ theories [12-28]. Some theories are second-order, like general relativity, while others are of at least

\footnotetext{
*yiannis.papayiannopoulos@gmail.com

svasil@academyofathens.gr

jdb34@hermes.cam.ac.uk

§anpaliat@phys.uoa.gr
}

fourth order. In the context of modified gravity, the field equations form a system of nonlinear ordinary differential equations which may not be integrable. In general, the role of integrability in any dynamical problem is to provide the necessary conditions to compute its solution. Obviously, the latter achievement is very important in cosmology. For example, one of the main problems that integrability solves is the determination of the initial conditions in cosmological simulations. Various methods have been proposed in order to study the integrability of dynamical systems. In Liouville's integrability approach to (classical) Hamiltonian systems, there is a point transformation for which the action is determined by the method of separation of variables. This is equivalent with the existence of a second conservation law which defines a support manifold such that intersects the phase space volume. However, this is not the only possibility, since the Hamilton-Jacobi can be solved explicitly without the existence of a coordinate system that leads to a separation of variables. In the second case, the conservation law provides a Lie-supported manifold for the dynamical system [29].

Conservation laws provide sufficient constraints to solve the Hamilton-Jacobi equation and they are related directly to the existence of transformations ("symmetries") which maintain dynamical invariance. Another way to study the integrability of a dynamical system is by the method of singularity analysis in which a necessary condition for success is the existence of a singular solution. Singularity analysis has been performed in various gravitational studies, and it can be used to determine important information about the evolution of the system close to a singularity [30-38]. 
In this work, we are interested in algebraic integrability in the sense that we will search for those $f(R)$-models for which the polynomial in the momentum conservation law exists and can be used to write the field equations as a system of two first-order differential equations. In order to determine the conservation laws, we use Noether's theorems. Specifically, from the first theorem we find the necessary conditions that the $f(R)$ theory needs to obey in order for there to exist a (generalized) symmetry vector, while from the second theorem the conservation law is determined. This is an analogue of the Ovsiannikov classification of the nonlinear heat equation [39] which has been applied in various gravitational theories and has led to new integrable models (see [40-55] and references therein). Noether's Theorem is the main mathematical tool that we use in this study and specifically we select to work within the framework of the so-called "contact symmetries" [56]. We extend our previous works $[57,58]$ to complete the classification of the integrable models in $f(R)$ gravity $[59,60]$. The plan of the paper is as follows.

In Sec. II, we briefly present the main points of $f(R)$ modified gravity with an ideal gas. Next, we derive the field equations and we discuss the minisuperspace Lagrangian in the context of the classical Hamiltonian formalism. Sections III and IV then present the main results of our analysis, namely we identify those families of $f(R)$ models for which the field equations admit extra local conservation laws and so form integrable dynamical systems. Finally, our conclusions are presented in Sec. V.

\section{THE GRAVITATIONAL FIELD EQUATIONS}

In this section, we introduce the main ingredients of $f(R)$ gravity. Specifically, the modified Einstein-Hilbert action of $f(R)$ gravity is

$$
S=\int d x^{4} \sqrt{-g}\left[\frac{1}{2 k} f(R)+L_{m}\right],
$$

where $R$ is the Ricci scalar, $g$ is the determinant of the metric tensor, $k \equiv 8 \pi G$ and $L_{m}$ is the Lagrangian function for the matter source. Varying $S$ with respect to the metric, we obtain the gravitational field equations

$$
f^{\prime} R_{\mu \nu}-\frac{1}{2} f g_{\mu \nu}-\left(\nabla_{\mu} \nabla_{\nu}-g_{\mu \nu} \nabla_{\sigma} \nabla^{\sigma}\right) f^{\prime}=k T_{\mu \nu},
$$

where $f^{\prime}(R)=\frac{d f}{d R}, R_{\mu \nu}$ is the Ricci tensor, and $T_{\mu \nu}=\frac{\partial L_{m}}{\partial g^{\prime \prime}}$ is the energy-momentum tensor for the matter source. It is interesting to see that the field equations can be cast in the following form

$$
R_{\mu \nu}-\frac{1}{2} R g_{\mu \nu}=k_{\mathrm{eff}}\left(T_{\mu \nu}+T_{\mu \nu}^{f(R)}\right),
$$

where $k_{\mathrm{eff}}=\frac{k}{f^{\prime}(R)}$. Also, the quantity $T_{\mu \nu}^{f(R)}$ can be viewed as the effective energy-momentum tensor of the modifications of the Einstein-Hilbert action, and is expressed as

$$
T_{\mu \nu}^{f(R)}=\left(\nabla_{\mu} \nabla_{\nu}-g_{\mu \nu} \nabla_{\sigma} \nabla^{\sigma}\right) f^{\prime}+\frac{1}{2}\left(f-R f^{\prime}\right) g_{\mu \nu} .
$$

Of course, for $f(R)=R$ the above formulas reduce to those of GR.

Now, assuming a spatially flat Friedmann-LemaîtreRobertson-Walker (FLRW) metric,

$$
d s^{2}=-d t^{2}+a^{2}(t)\left(d x^{2}+d y^{2}+d z^{2}\right),
$$

the field equations (2) give the modified Friedmann's equations:

$$
3 f^{\prime} H^{2}=k \rho_{m}+\frac{f^{\prime} R-f}{2}-3 H f^{\prime \prime} \dot{R},
$$

and

$$
\begin{aligned}
2 f^{\prime} \dot{H}+3 f^{\prime} H^{2}= & -2 H f^{\prime \prime} \dot{R}-\left(f^{\prime \prime \prime} \dot{R}^{2}+f^{\prime \prime} \ddot{R}\right) \\
& -\frac{f-R f^{\prime}}{2}-k p_{m},
\end{aligned}
$$

where $a(t)$ is the scale factor, $H=\dot{a} / a$ is the Hubble parameter $^{1}$ and $R=6\left[\frac{\ddot{a}}{a}+\left(\frac{\dot{a}}{a}\right)^{2}\right]$ in which the dot denotes derivative with respect to the comoving proper time, $t$. Notice that $\rho_{m}=T_{\mu \nu} u^{\mu} u^{\nu}$ and $p_{m}=T_{\mu \nu}\left(g^{\mu \nu}+u^{\mu} u^{\nu}\right)$ are the energy density and pressure of the matter source, where $u^{\mu}$ is the normalized four-velocity vector. For the equation of state of the cosmic matter, we use $p_{m}=w_{m} \rho_{m}$ which corresponds to an ideal gas, namely with $w_{m}=$ $\gamma-1=$ const, and $w_{m} \in[0,1]$ (or $\gamma \in[1,2]$ ). Utilizing the conservation law $T_{; \nu}^{\mu \nu}=0$ it is easy to show that $\rho_{m}$ evolves as $\rho_{m}=\rho_{m 0} a^{-3 \gamma}$, where $\rho_{m 0}$ is the corresponding density at the present time $\left(a=a_{0}=1\right)$. It is worth noting that for dominant relativistic matter we have $\gamma=4 / 3$ (when $\left.w_{m}=1 / 3\right)$, while in the case of pressureless matter we get $\gamma=1\left(w_{m}=0\right)$.

If we focus on the first Friedmann equation (6), then we can introduce an effective dark energy sector of (modified) gravitational origin. Indeed, the dark energy density and pressure are rewritten as

$$
\begin{gathered}
\rho_{f}=\frac{f^{\prime} R-f}{2}-3 H f^{\prime \prime} \dot{R}, \\
p_{f}=2 H f^{\prime \prime} \dot{R}+\left(f^{\prime \prime \prime} \dot{R}^{2}+f^{\prime \prime} \ddot{R}\right)+\frac{f-R f^{\prime}}{2},
\end{gathered}
$$

while the effective equation of state (EoS) parameter $w_{f}=\frac{p_{f}}{\rho_{f}}$ is given by $[6,7,43]$,

$$
w_{f}=-\frac{\left(f-R f^{\prime}\right)+4 H f^{\prime \prime} \dot{R}+2\left(f^{\prime \prime \prime} \dot{R}^{2}+f^{\prime \prime} \ddot{R}\right)}{\left(f-R f^{\prime}\right)+6 H f^{\prime \prime} \dot{R}} .
$$

\footnotetext{
${ }^{1}$ Recall that for an arbitrary lapse function $N(t)$ in the line element (5) the Hubble function is defined as $H=\frac{\dot{a}}{N a}$.
} 


\section{A. Minisuperspace Lagrangian}

From the technical point of view it will help our analysis to insert the Lagrange multiplier [61-63] $\lambda$ in the action (1) as follows

$$
\begin{aligned}
S= & \int d x^{4} \sqrt{-g}\left[f(R)-\lambda\left[R-6\left(\frac{\ddot{a}}{a}+\left(\frac{\dot{a}}{a}\right)^{2}\right)\right]\right. \\
& \left.+2 \rho_{m 0} a^{-3(\gamma-1)}\right],
\end{aligned}
$$

where we have set $\sqrt{-g} L_{m}=\rho_{m 0} a^{-3(\gamma-1)}$ and $k=$ $8 \pi G \equiv 1$. Now, if we vary the action with respect to $R$ $\left(\frac{\partial S}{\partial R}=0\right)$ we find $\lambda=f^{\prime}(R)$. Therefore, using the latter condition it is straightforward to obtain the overall Lagrangian of the current dynamical problem, namely

$$
\begin{aligned}
L(a, \dot{a}, R, \dot{R})= & 6 a f^{\prime} \dot{a}^{2}+6 a^{2} f^{\prime \prime} \dot{a} \dot{R}+a^{3}\left(f^{\prime} R-f\right) \\
& +2 \rho_{m 0} a^{-3(\gamma-1)} .
\end{aligned}
$$

One may check that, with the aid of Eq. (12), the EulerLagrange equations provide Eq. (7) and the definition of the Ricci scalar respectively. Furthermore, introducing a lapse function $N$ in the FLRW metric (5) the total Lagrangian becomes

$$
\begin{aligned}
L(N, a, \dot{a}, R, \dot{R})= & \frac{1}{N}\left(6 a f^{\prime} \dot{a}^{2}+6 a^{2} f^{\prime \prime} \dot{a} \dot{R}\right) \\
& +N a^{3}\left(f^{\prime} R-f\right)+2 \rho_{m 0} N a^{-3(\gamma-1)},
\end{aligned}
$$

The fact that the Lagrangian is independent from derivatives of $N$ implies that the corresponding Hessian vanishes and so the system is singular. Indeed, the first Friedmann equation (6) is the constraint equation, namely $\frac{\partial L}{\partial N}=0$, and it plays a central role in the Hamiltonian formalism as well as in the quantization of gravity. Moreover, without loss of generality we assume $N=N(a, R)$ which means that the constraint equation (6) can be viewed as a conservation law of the field equations. Lastly, we stress that based on the effective gravitational parameter $k_{\text {eff }}=\frac{k}{f^{\prime}(R)} \equiv \frac{1}{f^{\prime}(R)}$ we can provide the scalar-tensor representation of the $f(R)$ gravity which is equivalent to that of Brans-Dicke theory in the form of O'Hanlon [64]. In particular, considering an effective scalar field such as $\phi=f^{\prime}(R)$ the action integral (11) takes the following form

$$
S=\int d x^{4} \sqrt{-g}\left[\phi R+V(\phi)-2 \rho_{m 0} a^{-3(\gamma-1)}\right]
$$

with

$$
\begin{aligned}
L(N, a, \dot{a}, \phi, \dot{\phi})= & \frac{1}{N}\left(6 a \phi \dot{a}^{2}+6 a^{2} \dot{a} \dot{\phi}\right)+N a^{3} V(\phi) \\
& +2 \rho_{m 0} N a^{-3(\gamma-1)} .
\end{aligned}
$$

where we have set $V(\phi)=f^{\prime} R-f$. Obviously, this result corroborates, in the effective scalar field language, the transit of the $f(R)$ theory into the scalar-tensor regime which is a second-order theory [5].

The scalar field description of $f(R)$ gravity is useful because the order of the theory is reduced, while at the same time the number of the dependent variables is increased. Moreover, with the aid of the scalar field description one can use the Lagrange multiplier towards extracting a classical pointlike Lagrangian, which can be used in order to write the corresponding Hamiltonian and thus to derive the field equations $[65,66]$. Therefore, utilizing the latter mathematical treatment we can apply the known results of analytical mechanics in order to study the integrability of our dynamical system [67]. As an example, it is well known in analytical mechanics that the Legendre transformation relates the second-order Euler-Lagrange equations with the first-order Hamilton's equations, while the solutions and the integrability survive through the transformation. Following a similar ideology, one may easily understand the role of the transformation $\phi=f^{\prime}(R)$ in $f(R)$ gravity, namely it decreases the order of the theory from four to two $([61-63,65,66])$, while the solution trajectories remain invariant.

In addition, there is a conformal equivalence between $f(R)$. gravity theories and general relativity with a scalar field. The former theories are fourth order in the metric variables and the latter are second order in the effective scalar field, which is determined by the logarithm of the scalar curvature, $R$. From the point of view of the initial value problem, the general solution of general relativity plus a scalar field is determined by six independently arbitrary functions of the three spatial variables on a hypersurface of constant time, whereas the general solution of $f(R)$ gravity in vacuum is determined by 16 arbitrary spatial functions $[68,69]$. However, if the metric is specialized to the zero curvature Friedmann model then no free functions are required in either case. Likewise, all vacuum or trace-free fluid solutions of general relativity are also particular solutions of $f(R)$ gravity theories if $f(0)=0$ and are characterized by the same number of free spatial functions in both theories.

\section{Hamilton's equations}

Using the standard Hamiltonian approach, and with the aid of Eq. (15), we calculate the canonical momenta

$$
N p_{a}=12 a \phi \dot{a}+6 a^{2} \dot{\phi}, \quad N p_{\phi}=6 a^{2} \dot{a},
$$

so the Hamiltonian function becomes 


$$
\mathcal{H}=N\left[\frac{p_{a} p_{\phi}}{6 a^{2}}-\frac{\phi p_{\phi}^{2}}{6 a^{3}}-a^{3} V(\phi)-2 \rho_{m 0} a^{-3(\gamma-1)}\right],
$$

where the field equations of the previous section are those of Hamilton's equations and the constraint $\mathcal{H}=0$. In order to proceed with the dynamical analysis, we need to introduce the functional form of the lapse function $N$. Without loss of generality and in order to simplify the field equations we prefer to utilize $N=a^{3(\gamma-1)}$ and so the equations of motion reduce to the following system:

$$
\begin{gathered}
\dot{a}=\frac{a^{3 \gamma-5}}{6} p_{\phi}, \quad \dot{\phi}=\frac{a^{3 \gamma-5}}{6}\left(p_{a}-\frac{\phi}{a} p_{\phi}\right), \\
\dot{p}_{\phi}=\frac{a^{3 \gamma-6}}{6} p_{\phi}^{2}+a^{3 \gamma} V_{, \phi},
\end{gathered}
$$

and

$$
\begin{aligned}
\dot{p}_{a}= & -\frac{a^{3 \gamma-7}}{6} p_{\phi}\left((3 \gamma-5) a p_{a}-3(\gamma-2) \phi p_{\phi}\right) \\
& +3 \gamma a^{3 \gamma-1} V(\phi) .
\end{aligned}
$$

Dynamically speaking, these form a two-dimensional autonomous Hamiltonian system. Except for the nominal conservation law, namely $\mathcal{H}=0$, it is essential to determine a second conservation law in order to characterize the dynamical system as integrable. However, the existence of a second conservation law depends strongly on the functional form of the effective potential $V(\phi)$, or equivalently, on the form of $f(R)$. It is important to mention that our analysis and the solutions that we find below can be transformed for any other lapse function $N(t)$, for more details we refer the reader to the discussion in [70].

In the rest of the paper, we will study a family of effective potentials for which the corresponding field equations are integrable.

\section{ANALYTIC SOLUTIONS}

The fact that the field equations in $f(R)$ gravity form a canonical Hamiltonian system implies that we can use the basic tools of classical mechanics in order to investigate the integrability of the system. Specifically, we are interested in those cases where the field equations are invariant under generalized symmetries and are linear in the momentum: the so-called contact symmetries. These symmetries provide quadratic conservation laws in the momentum and they can be determined by using Noether's second theorem. In the Appendix, we provide the basic mathematical tools of the method used here. More details regarding the application of generalized symmetries in modified theories of gravity can be found in Refs. [57,58,60].

The application of the generalized symmetries provides the following general form of the effective potential

$$
V(\phi)=V_{1} \phi^{P}+V_{2} \phi^{Q}
$$

where the constants $P, Q$ are functions of the barotropic parameter $\gamma$. Obviously, this potential describes a large body of $f(R)$ models. In what follows, we discuss the different cases for the parameters $P, Q$, which follow from the symmetry conditions in order for the field equations to admit extra conservation laws.

\section{A. Model A}

$$
\begin{aligned}
& \text { For }(P, Q)=\left(0,-\frac{1}{2}\right) \text { the potential is } \\
& \qquad V_{A}(\phi)=V_{1}+V_{2} \phi^{-\frac{1}{2}},
\end{aligned}
$$

and the field equations (18)-(20) admit the following extra conservation law:

$$
I_{A}=6 a^{9-6 \gamma} \dot{a}[(3 \gamma-4) \phi \dot{a}+a \dot{\phi}]-a^{5}\left(\frac{3}{5} V_{1} \gamma+V_{2} \phi^{-\frac{1}{2}}\right) \text {, }
$$

for $\gamma \neq \frac{5}{3}$, or

$$
\begin{aligned}
\bar{I}_{A}= & 30 a^{-1} \dot{a}[\phi(\ln a-1) \dot{a}+\dot{\phi} a \ln a] \\
& -5 a^{5} \ln a\left(V_{1}+V_{2} \phi^{-\frac{1}{2}}\right)+V_{1} a^{5}
\end{aligned}
$$

for $\gamma=5 / 3$. At this point we should mention that the above effective potential (22) has been studied in [60] and it corresponds to $f(R) \simeq R^{\frac{1}{3}}-f_{0}$ gravity. However, the conservation law (23) is more general with respect to that of [60], since in the latter article only the case of a dust fluid $(\gamma=1)$ has been used.

Performing the transformation $a=x^{-\frac{1}{3 \gamma-5}}, \phi=y x^{\frac{1}{3 \gamma-5}}$, for $\gamma \neq \frac{5}{3}$ the constraint equation and the conservation law $I_{A}$ are written as

$\frac{(5-3 \gamma)}{6} p_{x} p_{y}-V_{1} x^{\frac{3 \gamma}{5-3 \gamma}}-V_{2} x^{\frac{6 \gamma+1}{2(5-3 \gamma)}} y^{-\frac{1}{2}}-2 \rho_{m 0}=0$

and

$\frac{(5-3 \gamma)}{6} p_{y}\left(y p_{y}-x p_{x}\right)+\frac{3}{5} \gamma V_{1} x^{\frac{5}{5-3 \gamma}}+V_{2} x^{\frac{11}{2(5-3 \gamma)}} y^{-\frac{1}{2}}-I_{A}=0$,

with

$$
\dot{x}=\frac{5-3 \gamma}{6} p_{y}, \quad \dot{y}=\frac{5-3 \gamma}{6} p_{x} .
$$

Hence, from Eqs. (25), (26), the action $S(x, y)$ can be determined and the field equations are reduced to those of (27) where 


$$
p_{x}=\frac{\partial S(x, y)}{\partial x} \quad \text { and } \quad p_{y}=\frac{\partial S(x, y)}{\partial y} .
$$

We find after some calculations that in the cases of dust $(\gamma=1)$ and relativistic matter $(\gamma=4 / 3)$ the corresponding actions $S(x, y)$ are given by

$$
\begin{aligned}
S(x, y)= & -\frac{2 \sqrt{15}}{5} \sqrt{y\left(5 I_{A}+10 \rho_{m 0} x+2 V_{1} x^{\frac{5}{2}}\right)} \\
& -\int \frac{\sqrt{15} V_{2} x^{\frac{7}{4}} d x}{\sqrt{\left(5 I_{A}+10 \rho_{m 0} x+2 V_{1} x^{\frac{5}{2}}\right)}} \text { for } \gamma=1
\end{aligned}
$$

and

$$
\begin{aligned}
S(x, y)= & -\frac{2 \sqrt{30}}{5} \sqrt{y\left(5 I_{A}+10 \rho_{m 0} x+V_{1} x^{5}\right)} \\
& -\int \frac{\sqrt{30} V_{2} x^{\frac{9}{2}} d x}{\sqrt{\left(5 I_{A}+10 \rho_{m 0} x+V_{1} x^{5}\right)}}, \text { for } \gamma=4 / 3 .
\end{aligned}
$$

\section{Special case}

In order to complete the analysis of the potential (22), we consider the case of $\gamma=\frac{5}{3}$. Utilizing the coordinate transformation $a=e^{u}, \phi=v e^{-u}$ the constraint equation and the conservation law $\bar{I}_{A}$ become

$$
\frac{p_{u} p_{v}}{6}-V_{1} e^{5 u}-e^{\frac{11}{2} u} v^{-\frac{1}{2}}-2 \rho_{m 0}=0,
$$

and

$\frac{5}{6} p_{v}\left(u p_{u}-v p_{v}\right)+V_{1} e^{5 u}(1-5 u)-5 V_{2} e^{\frac{11}{2} u} u v^{-\frac{1}{2}}-\bar{I}_{A}=0$.

Therefore, the action is calculated to be

$$
\begin{aligned}
S(u, v)= & -\frac{2 \sqrt{30}}{5} \sqrt{v\left(10 u \rho_{m 0}+V_{1} e^{5 u}-\bar{I}_{A}\right)} \\
& -\sqrt{30} V_{2} \int \frac{e^{\frac{11}{2} u}}{\sqrt{\left(10 u \rho_{m 0}+V_{1} e^{5 u}-\bar{I}_{A}\right)}} d u
\end{aligned}
$$

while the field equations are reduced to the twodimensional system,

$$
\begin{aligned}
& \dot{u}=-\frac{\sqrt{30}}{v} \sqrt{\left(10 u \rho_{m 0}+V_{1} e^{5 u}-\bar{I}_{A}\right)}, \\
& \dot{v}=\frac{\sqrt{30}}{6} \frac{V_{1} v+2 \rho_{m 0} v+\sqrt{v} V_{2} e^{\frac{11}{2} u}}{\sqrt{\left(10 u \rho_{m 0}+V_{1} e^{5 u}-\bar{I}_{A}\right) v}} .
\end{aligned}
$$

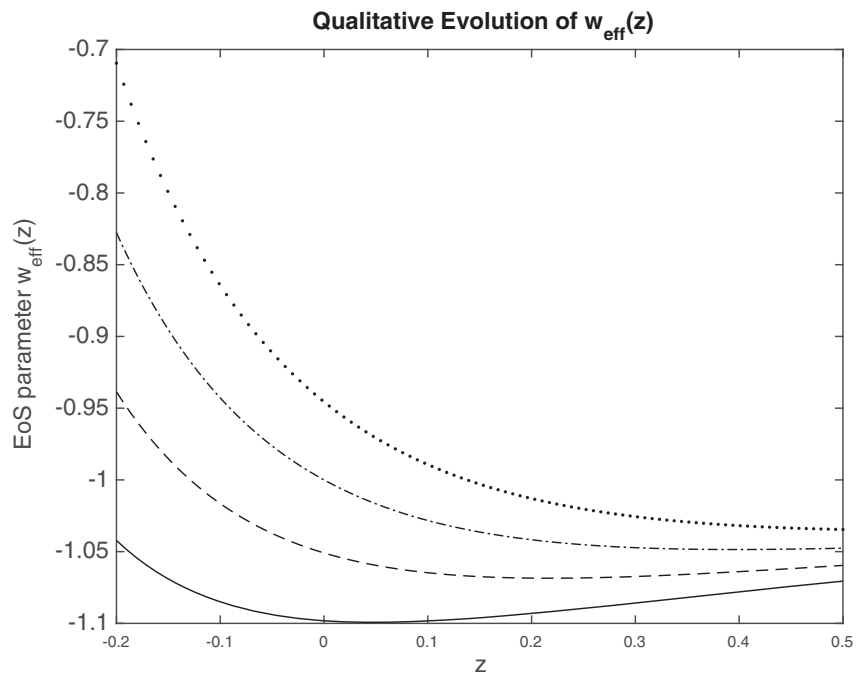

FIG. 1. Qualitative evolution of the equation of state parameter (39). For the plot we selected $V_{2}=2$. The solid line is for $V_{1}=0.65$, the dash-dash line for $V_{1}=0.70$ while the dash-dot and dot-dot liens are for $V_{1}=0.75$ and $V_{1}=0.80$ respectively.

For large values of $u$, that is in the late universe, this system approximates to

$$
\dot{u} \simeq-\frac{\sqrt{30}}{v} \sqrt{\left(V_{1} e^{5 u}\right)}, \quad \dot{v} \simeq \frac{\sqrt{30}}{6 \sqrt{V_{1}}} V_{2} e^{3 u},
$$

which means that $\int \frac{d v}{v}=-\left(\frac{V_{2}}{6 V_{1}}\right) \int e^{\frac{1}{2} u} d u$ and $\ln (v)=$ $\left(-\frac{V_{2}}{3 V_{1}}\right) e^{\frac{1}{2} u}$. In this case, after some calculations, we find

$$
\phi(a) \simeq-\frac{1}{9} \frac{V_{2}}{V_{1}} a^{\frac{5}{2}}+\phi_{0}
$$

Lastly, the Hubble function for large values of $a$ takes the form

$$
H \simeq a^{-\frac{1}{4}} \exp \left(\frac{V_{1} V_{2}}{3} \sqrt{a}\right)
$$

Also, the effective equation of state (EoS) parameter is found to be

$$
w_{\mathrm{eff}}(a)=-1-\frac{\sqrt{30 V_{1}}}{18}\left(3-2 V_{2} V_{1} \sqrt{a}\right) a^{\frac{11}{4}}
$$

Notice that, in fig. 1, we present the redshift evolution of the latter EoS parameter.

\section{B. Model B}

In this class of models, the constants $P$ and $Q$ in the effective potential (21) have the following simple expressions 


$$
P=-\frac{\gamma}{\gamma-2}, \quad Q=-\frac{3 \gamma-10}{3 \gamma-4}
$$

Obviously, the prohibited values $\gamma \neq 4 / 3$ and $\gamma \neq 2$ imply that in this case the cosmic fluid cannot be that of radiation or stiff matter. These two special cases need to be studied separately.

Now, the conservation law is

$$
I_{B}=a^{10-6 \gamma}[(3 \gamma-4) \phi \dot{a}+a \dot{\phi}]^{2}-\frac{2(3 \gamma-5)}{3} V_{2} a^{6} \phi^{\frac{6}{3 \gamma-4}}
$$

for $\gamma \neq \frac{5}{3}$. At this point we use the normal coordinates $(x, y)=\left(r e^{\theta}, r e^{-\theta}\right)$, where the pair $(x, y)$ is given by $a=x^{-\frac{1}{3 \gamma-5}}, \phi=y x^{\frac{1}{3 \gamma-5}}$, for $\gamma \neq \frac{5}{3}$. It is worth noting that in the new coordinates the conservation law is that of the Lewis invariant which means that the dynamical system admits as a conservation law $^{2}$ the Lewis-invariant [75]. Within this framework, we define the constraint equation and the conservation law, $I_{B}$, by

$$
\begin{gathered}
(3 \gamma-5)\left(\frac{p_{r}^{2}}{24}+\frac{p_{\theta}^{2}}{24 r^{2}}\right)-V_{1} r^{-\frac{2 \gamma}{\gamma-2}}-\frac{V_{2}}{r^{2}} e^{\frac{12 \theta}{4-3 \gamma}}-2 \rho_{m 0}=0 \\
\frac{p_{\theta}^{2}}{36}+2 V_{2}\left(\frac{5}{3}-\gamma\right) e^{\frac{12 \theta}{4-3 \gamma}}-I_{B}=0
\end{gathered}
$$

with

$$
\dot{r}=\frac{5-3 \gamma}{12} p_{r}, \quad \dot{\theta}=-\frac{3 \gamma-5}{12 r^{2}} p_{\theta}
$$

After some simple algebra, we obtain the corresponding action $S(r, \theta)$

$$
\begin{gathered}
S(r, \theta)=\int \sqrt{72\left(\left(\gamma-\frac{5}{3}\right) V_{2} e^{\frac{-12 \theta}{-4+3 \gamma}}-\bar{I}_{B}\right)} d \theta+ \\
-\int \sqrt{72\left(-2 \rho_{m 0}\left(\gamma-\frac{5}{3}\right)-\left(\gamma-\frac{5}{3}\right) V_{1} r^{-\frac{2 \gamma}{-2+\gamma}}-\frac{1}{72} \bar{I}_{B} r^{-2}\right)} d r .
\end{gathered}
$$

Using the conservation law, we find that the constraint equation reads

\footnotetext{
${ }^{2}$ For more applications of the Ermakov-Pinney system in gravitational theories and cosmology, see [71-74].
}

$$
\frac{p_{r}^{2}}{24}-(3 \gamma-5) V_{1} r^{-\frac{2 \gamma}{\gamma-2}}+\frac{3 I_{B}}{r^{2}}=2(3 \gamma-5) \rho_{m 0}
$$

Inserting the expression $\dot{r}=\frac{5-3 \gamma}{12} p_{r}$ into Eq. (47), we have

$$
\frac{(3 \gamma-5) \dot{r}^{2}}{3456}-V_{1} r^{-\frac{2 \gamma}{\gamma-2}}+\frac{3 I_{B}}{(3 \gamma-5) r^{2}}=2 \rho_{m 0}
$$

Interestingly, in the case of $\gamma=0\left(w_{m}=-1\right)$, the above equation becomes

$$
\frac{5}{3456} \dot{r}^{2}+\frac{3 I_{B}}{5} r^{-2}=V_{1}-2 \rho_{m 0}=C\left(\rho_{m 0}, V_{1}\right) .
$$

a solution of which is

$$
\begin{aligned}
r^{2}(t) & =\frac{3}{5 C}\left(c+I_{B}\right)+\frac{3}{5}\left(-c_{1} \bar{C} t+\bar{C} t^{2}\right) \\
& =r_{0}+\frac{3}{5}\left(-c_{1} \bar{C} t+\bar{C} t^{2}\right)
\end{aligned}
$$

where $c_{1}$ is an integration constant, $C$ is a constant that depends from $\left(\rho_{m 0}, V_{1}\right)$ and $\bar{C}=1152 C$. Hence, this analytical solution behaves as $r \simeq t$ and $r \simeq t^{2}$ for small and large values of $t$, respectively.

Moreover, in the case of $\bar{I}_{B}=0$, it follows that $e^{\theta(t)}=\Theta_{0}\left(\int \frac{d t}{r(t)^{2}}\right)^{\lambda(\gamma)}$, which implies that for small or large values of $t$ we have $a(t) \simeq t^{\lambda_{1}(\gamma)}(\ln t)^{\lambda_{2}(\gamma)}$ and $a(t) \simeq$ $t^{\lambda_{1}(\gamma)} e^{t_{3}(\gamma)}$, where $\lambda(\gamma)$ are constants related to the barotropic index $\gamma$. Recall that these solutions are expressed in the time in which the lapse function is $N(t)=a^{3(\gamma-1)}$.

Below, we will consider some special cases, namely $\gamma=5 / 3,4 / 3$ and 2 .

\section{Special case $\gamma=5 / 3$}

Here we consider $\gamma=\frac{5}{3}$; hence, from Eq. (40), we have $P=Q=5$ and the effective potential is given by $V(\phi) \propto \phi^{5}$. The coordinates that we use here are those of Sec. III A 1, namely, $(u, v)$. In this context, we obtain the constraint equation and the conservation law $I_{B}$ :

$$
\begin{gathered}
\frac{p_{u} p_{v}}{6}+\left(V_{1}+V_{2}\right) v^{5}+2 \rho_{m 0}=0, \\
\frac{p_{u}^{2}}{36}-I_{B}=0 .
\end{gathered}
$$

with $\dot{v}=\frac{1}{6} p_{u}, \dot{u}=\frac{1}{6} p_{v}$. Here, the action takes the form

$$
S(u, v)=6 \sqrt{I_{B}} u+\frac{2\left|\rho_{m 0}\right|}{\sqrt{I_{B}}} v-\frac{\left(V_{1}+V_{2}\right)}{36 \sqrt{I_{B}}} v^{6}
$$

that is, 


$$
\dot{v}=\sqrt{I_{B}} \quad \text { and } \quad \dot{u}=\frac{2\left|\rho_{m 0}\right|}{6 \sqrt{I_{B}}}-\frac{\left(V_{1}+V_{2}\right)}{36 \sqrt{I_{B}}} v^{5}
$$

or equivalently

$$
v(t)=\sqrt{I_{B}} t, \quad \dot{u}=\frac{\left|\rho_{m 0}\right|}{6 \sqrt{I_{B}}} t-\frac{\left(V_{1}+V_{2}\right)\left(I_{B}\right)^{2}}{180} t^{6} .
$$

Notice, that the scale factor of the universe is approximated by $a(t)=e^{u} \simeq e^{\frac{\left|\rho_{m 0}\right|}{6 \sqrt{I_{B} t^{2}}}}$ and $a(t)=e^{-\frac{\left(V_{1}+V_{2}\right)\left(I_{B}\right)^{2}}{180} t^{7}}$ for small and large values of $t$, respectively, where for the latter case we need to have $V_{1}+V_{2}<0$. Furthermore, the expression for the Hubble function at small values of $t$ is found to be

$$
H(a) \simeq a^{-12}(\ln a)^{1 / 2}
$$

while for large values of $t$ we get

$$
H(a) \simeq-\left(V_{1}+V_{2}\right) a^{4}(\ln a)^{6 / 7} .
$$

\section{Radiation fluid}

As we have already discussed, Model B does not exist for $\gamma=\frac{4}{3}\left(w_{m}=1 / 3\right)$. In this case, the potential is

$$
V(\phi)=V_{1} \phi^{2}
$$

while the corresponding conservation law generated by the same Killing tensor becomes

$$
I_{B}=a^{4} \dot{\phi}^{2}
$$

Performing the transformation $a=r(t)[\cosh \vartheta(t)+$ $\sinh \vartheta(t)], \phi=r(t)[\cosh \vartheta(t)-\sinh \vartheta(t)]$, we find that the constraint equation and the conservation law $I_{B}^{(r)}$ are written as

$$
\begin{gathered}
V_{1} r^{4}+6 \dot{r}^{2}-2 \rho_{0}+6 r^{2} \dot{\vartheta}^{2}=0 \\
6 r^{4} \dot{\vartheta}^{2}-I_{B}=0
\end{gathered}
$$

from which we obtain directly

$$
\dot{\vartheta}^{2}=\frac{I_{B}}{6 r^{4}}, \quad V_{1} r^{4}+6 \dot{r}^{2}-2 \rho_{0}+\frac{I_{B}}{r^{2}}=0 .
$$

Solving the above set of equations, we can define the quantities $r(t)$ and $\theta(t)$. For example, if we assume for simplicity that $\rho_{0}=I_{B}=0$, then we find

$$
r(t)= \pm \frac{6}{\sqrt{-6 V_{1}}\left(c_{1}-t\right)}, \quad \vartheta(t)=c_{2}
$$

and thus $a(t) \simeq \frac{6}{\sqrt{-6 V_{1}}\left(c_{1}-t\right)}, \phi(t) \simeq \pm \frac{6}{\sqrt{-6 V_{1}}\left(c_{1}-t\right)}$. In this case, the Hubble function can now be directly calculated
$(N=a)$ and shown to be a constant, that is $(H(a))^{2}=$ $\frac{\dot{a}}{N a}=\frac{\left|V_{1}\right|}{6}$, which is the de Sitter solution. That is an expected result since the current potential corresponds to the $f(R)=R^{2}$ theory.

\section{Model C}

The third model that we find is that for which the constants $P$ and $Q$ are expressed in terms of the barotropic index by

$$
P=\frac{3 \gamma+5}{2}, \quad Q=3 \gamma .
$$

For this model the corresponding conservation law is calculated to be

$$
\begin{aligned}
I_{C}= & (3 \gamma-4) a^{5-3 \gamma} \phi^{2} \dot{a}^{2}+3(\gamma-1) a^{6-3 \gamma} \phi \dot{a} \dot{\phi}+a^{7-3 \gamma} \dot{\phi}^{2} \\
& +-\frac{(3 \gamma-5)}{6} V_{1} a^{3 \gamma+1} \phi^{\frac{7+3 \gamma}{2}}-\frac{3 \gamma-5}{1+3 \gamma} \gamma V_{2}(a \phi)^{3 \gamma+1}
\end{aligned}
$$

Using the transformation $a=z^{\frac{-2}{3 \gamma-5}}, \phi=w z^{\frac{2}{3 \gamma-5}}$, we write the constraint equation and the conservation law in the new coordinate system as

$$
\begin{aligned}
- & V_{2} w^{3 \gamma}-V_{1} \frac{w^{\frac{5+3 \gamma}{2}}}{z}+\frac{5-3 \gamma}{12 z} p_{w} p_{z}-4 \rho_{m 0}=0 \\
I_{C}= & \frac{w p_{z} p_{w}}{72 z}(5-3 \gamma)^{2}+\frac{p_{z}^{2}}{144}(5-3 \gamma)^{2}+\frac{V_{1}(5-3 \gamma)}{6 z} w^{\frac{7+3 \gamma}{2}} \\
& +\frac{V_{2} \gamma(5-3 \gamma)}{2(1+3 \gamma)} w^{1+3 \gamma}
\end{aligned}
$$

where now $\dot{z}=\frac{5-3 \gamma}{12 z} p_{w}$ and $\dot{w}=\frac{5-3 \gamma}{12 z} p_{z}$.

Following [29], we can see that the dynamical system is supported by a Lie surface and the solution of the Hamilton-Jacobi equation provides the action

$S(z, w)=z \sqrt{I c+\frac{24 V_{2}}{(5-3 \gamma)(3 \gamma+1)} w^{3 \gamma+1}+\frac{48 \rho_{m 0}}{5-3 \gamma} w}$

$$
+\int d w \frac{\frac{12 V_{1}}{5-3 \gamma} w^{\frac{5+3 \gamma}{2}}}{\sqrt{I c+\frac{24 V_{2}}{(5-3 \gamma)(3 \gamma+1)} w^{3 \gamma+1}+\frac{48 \rho_{m 0}}{5-3 \gamma} w}}
$$

where $\gamma \neq 5 / 3$.

\section{Special case $\gamma=5 / 3$}

Again, in the special case of $\gamma=\frac{5}{3}$, we utilize the coordinates of Sec. III A 1, $(u, v)$. Therefore, the constraint equation and the conservation law $I_{c}^{(u)}$ now take the form 


$$
\begin{gathered}
-p_{u} p_{v}+6\left(V_{1}+V_{2}\right) v^{5}+4 \rho_{m 0}=0 \\
\frac{p_{u}^{2}}{36}-I_{C}=0
\end{gathered}
$$

Now, we obtain

$$
\dot{v}=\sqrt{I_{c}^{(u)}} \quad \text { and } \quad \dot{u}=\frac{\rho_{m 0}}{9 \sqrt{I_{c}^{(u)}}}+\frac{\left(V_{1}+V_{2}\right)}{6 \sqrt{I_{c}^{(u)}}} v^{5},
$$

where $\dot{v}=\frac{1}{6} p_{u}, \dot{u}=\frac{1}{6} p_{v}$. Hence, we can write an analytic solution in closed-form, namely

$$
\begin{aligned}
& v(t)=\sqrt{I_{C}} t \text { and } \\
& u(t)=\frac{\rho_{m 0}}{9 \sqrt{I_{C}}} t+\frac{\left(V_{1}+V_{2}\right)\left(I_{C}\right)^{\frac{9}{2}}}{36} t^{6}+c,
\end{aligned}
$$

and thus we obtain

$$
a(t)=\exp \left(\frac{\rho_{m 0}}{9 \sqrt{I_{C}}} t+\frac{\left(V_{1}+V_{2}\right)\left(I_{C}\right)^{\frac{9}{2}}}{36} t^{6}\right)+c .
$$

For small values of $t$, we easily see that $a(t) \simeq$ $\exp \left(A_{0} t\right)+c$, while for large values of $t$ the scale factor becomes $a(t) \simeq \exp \left(A_{1} t^{6}\right)+c$. Using the expression of the Hubble function $H(a)=\frac{1}{N} \frac{\dot{a}}{a}$ where now $N=a^{2}$ we find

$$
H(a) \simeq a^{-\frac{3}{4}}-c a^{-\frac{7}{4}}
$$

for small values of $t$, and

$$
H(a) \simeq 6\left(A_{1}\right)^{\frac{1}{6}}(\ln (a-c))^{\frac{5}{6}}(a-c) a^{-\frac{7}{4}},
$$

for large values of the cosmic time. Finally, in this scenario the corresponding action is given by

$$
S(u, v)=I_{C}^{-\frac{1}{2}}\left[\left(V_{1}+V_{2}\right) v^{6}+24 v \rho_{m 0}+36 u I_{C}\right] .
$$

\section{QUINTESSENCE AND ACCELERATION FOR EMPTY SPACETIME}

The action integral of the O'Hanlon field $\phi$ in a fourdimensional empty spacetime is given from the following expression:

$$
S=\int d x^{4} \sqrt{-g}[\phi R-V(\phi)]
$$

Under the conformal transformation $\bar{g}_{i j}=\phi g_{i j}$, Eq. (76) becomes

$$
S_{M N}=\int d x^{4} \sqrt{-\bar{g}}\left[-\frac{\bar{R}}{2}+\frac{1}{2} \bar{g}^{i j} \nabla_{\mu} \psi \nabla_{\nu} \psi-e^{-\frac{2 \psi}{\sqrt{3}}} V(\psi)\right],
$$

where $\bar{R}$ now is the Ricci scalar of the conformal metric $\bar{g}_{i j}$ and $\psi$ is related with the field $\phi$ as

$$
\psi=\sqrt{3} \ln \phi \quad \text { or } \quad \phi=\exp \left(\frac{\sqrt{3}}{3} \psi\right) .
$$

The main difference between the fields $\phi$ and $\psi$ is that they are defined in different frames. Specifically, $\psi$ is minimally coupled scalar field, while $\phi$ is nonminimally coupled and defined in the Jordan frame.

In our case, with the aid of Eq. (78), the family of potentials provided by Eq. (21) is written in terms of $\psi$ as follows,

$$
\bar{V}(\psi)=V_{1} \exp (\alpha \psi)+V_{2} \exp (\alpha \psi),
$$

in which $\alpha=(P-2) / \sqrt{3}$ and $\beta=(Q-2) / \sqrt{3}$.

This class of potentials has been studied in the early universe in Ref. [76], and recently a special case of this family of potentials has been investigated in the constantroll inflationary regime in Ref. [77]. Barreiro et al. [76] have shown that Eq. (79) can describe various scaling solutions in the early universe which depend on the constants appearing in the exponential terms, namely $\alpha$ and $\beta=\frac{(Q-2)}{\sqrt{3}}$.As was discussed in [76], one of the coefficients $\alpha, \beta$ has to be greater than 5.5, (for example, we assume $|\alpha|>5.5$ ), in order for the theory to be in agreement with the constraints imposed by the primordial nucleosynthesis. Furthermore, in order to have a quintessence description with equation of state parameter that fits observations, the second free parameter is constrained by $\beta<0.8$. Moreover, for those values of the parameters where $\alpha \beta>0$, there exists a late-time attractor which describes and accelerated universe with equation of state parameter different from -1 . However, for $\alpha \beta<0$ the equation of state parameter of the late-time attractor has an oscillatory behavior until it mimics the cosmological constant.

In Fig. 2, we present the exponential rates of the potential (79) for the second family class of models. If we assume that $\gamma$ is to be the parameter of a barotropic fluid, that is, $\gamma \in[1,2)$ the vertical axis is constrained by the same bounds. In general, mathematically $\gamma$ can take any value except $\gamma \neq \frac{4}{3}$. However, close to the value $\gamma=\frac{4}{3}$, which corresponds to the limit where the matter source is radiation, we can see that our parameters are in agreements with the bounds that we discussed on the previous paragraph and specifically those arising when $\alpha \beta>0$. 


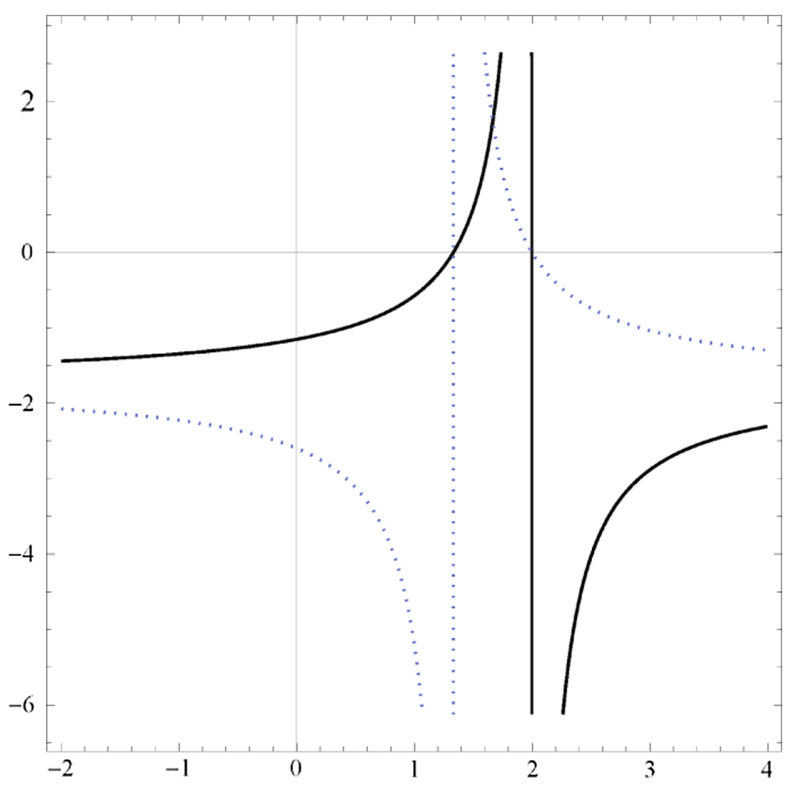

FIG. 2. Exponential rates of the early universe potential (79) for the family class of models B which corresponds to the ErmakovPinney system.

\section{CONCLUSIONS}

In this work, we have provided new analytical solutions for $f(R)$ gravity models which contain an ideal fluid in a spatially flat FLRW universe. In order to determine the $f(R)$ models which admit exact solutions, we applied the well known method of invariant transformations and, with the aid of Noether's second theorem, we constructed the corresponding conservation law which was used to solve the Hamilton-Jacobi equation. Then, this solution was applied in order to reduce the field equations to a system of two firstorder ordinary differential equations and express the solution in closed-form (where possible). It is interesting to mention that some of the current $f(R)$ models have been discussed in Ref. [59], and in the framework of Brans-Dicke gravity in Ref. [58]. However, in this work we found new cases of integrable $f(R)$ models. Therefore, in the context of spatially flat FLRW metrics, the combination of the present work with those of Paliathanasis et al. [59] and Papagianopoulos et al. [58] provides a complete study of the integrable $f(R)$ models that contain conservation laws that are quadratic in momentum. Finally, since the issue of frames are still open, namely if analytical solutions have to be interpreted either in the Einstein frame or in the Jordan frame, we have studied conformally related metrics and scalar fields in the framework of O'Hanlon's theory. In the Jordan frame, we determined the corresponding minimally coupled scalar field potential which corresponds to the integrable models that we found in the Einstein frame.

\section{ACKNOWLEDGMENTS}

S. B. acknowledges support by the Research Center for Astronomy of the Academy of Athens in the context of the program "Testing general relativity on cosmological scales" (Ref. number 200/872). A. P. was financially supported by FONDECYT Grants No. 3160121. A.P. thanks the University of Athens for the hospitality provided while part of this work was performed. J. D. B. acknowledges support from the Science and Technology Facilities Council (STFC) of the United Kingdom.

\section{APPENDIX: SYMMETRIES AND CONSERVATION LAWS}

In this appendix, we present the basic mathematical material which applied in this work towards determining the conservation law of the gravitational field equations.

Consider a differential function $F\left(x^{i}, u^{A}, u_{, i}^{A}, u_{, i j}^{A} \ldots\right)$ which is defined in the jet space $\left\{x^{i}, u^{A}, u_{, i}^{A}, u_{, i j}^{A}, \ldots\right\}$ where $x^{i}$ denotes the independent variables and $u^{A}$ are dependent variables. The differential function $F$ will be invariant under the infinitesimal transformation,

$$
\begin{gathered}
\bar{x}^{i}=x^{i}+\varepsilon \xi^{i}\left(x^{i}, u^{B}, u_{, i}^{B}, u_{, i j}^{B} \ldots\right), \\
\bar{u}^{A}=u^{A}+\varepsilon \eta^{A}\left(x^{i}, u^{B}, u_{, i}^{B}, u_{, i j}^{B} \ldots\right),
\end{gathered}
$$

if and only if $\iota F\left(x^{i}, u^{A}, u_{, i}^{A}, u_{, i j}^{A} \ldots\right)=\bar{F}\left(\bar{x}^{\bar{l}}, \bar{u}^{\bar{A}}, \bar{u}_{\overline{,}}^{\bar{A}}, \bar{u}_{, \bar{l}}^{\bar{A}} \ldots\right)$, which means that at every point the value of the differential function will be the same [78,79]. This is equivalent to the following mathematical expression,

$$
X^{[n]} F=\lambda F,
$$

where $X$ is the generator of infinitesimal transformation (A1)-(A2) defined as

$X=\xi^{i}\left(x^{i}, u^{B}, u_{, i}^{B}, u_{, i j}^{B} \ldots\right) \partial_{i}+\eta^{A}\left(x^{i}, u^{B}, u_{, i}^{B}, u_{, i j}^{B} \ldots\right) \partial_{u}$.

$X^{[n]}$ denotes its extension in the jet space $\left\{x^{i}, u^{A}\right.$, $\left.u_{, i}^{A}, u_{, i j}^{A}, \ldots\right\}$ and $\lambda=\lambda\left(x^{i}, u, u_{, i}, u_{, i j} \ldots\right)$ is a function which should determined. If there is a function $\lambda$ such that the condition (A3) holds then the generator $X$ is called a symmetry of the differential function.

The functional form of the generator (A4) defines the kind of symmetries. For instance, when $\xi^{i}, \eta^{A}$ are functions only of $\left\{x^{i}, u^{A}\right\}$ then $X$ is called a point symmetry, while if $\xi^{i}, \eta^{A}$ are linear in the first derivatives, $u_{, i}^{B}$, then $X$ is called contact symmetry. Of course, all the vector fields are Lie symmetries because we are dealing with local infinitesimal transformations.

There are various methods to construct conservation laws for differential functions/equations with the aid of the symmetry vectors. However, the simplest method is to apply Noether's theorems. The first of these states that if 
the action integral is invariant under the action of an infinitesimal transformation then the field equations are invariant. For Lagrangian functions of the form $\mathcal{L}\left(t, q^{i}, \dot{q}^{i}\right)$, which describe second-order differential equations, Noether's first theorem takes the form,

$$
X^{[1]} \mathcal{L}+\mathcal{L} \frac{d \xi}{d t}=\Phi
$$

where $\xi$ is the component of the generator $X$ in the direction of the independent variables and $\Phi=\Phi(t, q, \dot{q})$ is a boundary function.

In our discussion, the Lagrangian of the field equations has the form $\mathcal{L}=T-V$; that is, $\mathcal{L}\left(q^{k}, \dot{q}^{k}\right)=\frac{1}{2} \gamma_{i j} \dot{q}^{i} \dot{q}^{j}-V\left(q^{k}\right)$ where $\gamma_{i j}\left(q^{k}\right)$ is the minisuperspace metric. Therefore, with this family of Lagrangians, and for contact symmetries in which $X=K_{j}^{i}\left(t, q^{k}\right) \dot{q}^{i} \partial_{i}$, the Noether symmetry condition (A5) gives the condition

$$
K^{i j} V_{j}+\Phi_{, i}=0
$$

where $K_{i j}=K_{i j}\left(q^{k}\right)$ is a Killing tensor of the minisuperspace.

Finally, Noether's second theorem can be applied to write the explicit form of the corresponding conservation law, which for these types of contact symmetry takes the simple form, $I=K_{i j} \dot{q}^{j} \frac{\partial \mathcal{L}}{\partial \dot{q}^{i}}-\Phi$.
[1] H. Nariai and K. Tomita, Prog. Theor. Phys. 46, 776 (1971).

[2] A. A. Starobinsky, Phys. Lett. B 91, 99 (1980).

[3] H. A. Buchdahl, Mon. Not. R. Astron. Soc. 150, 1 (1970).

[4] J. D. Barrow and A. C. Ottewill, J. Phys. A 16, 2757 (1983).

[5] J. D. Barrow and S. Cotsakis, Phys. Lett. B 214, 515 (1988).

[6] T. P. Sotiriou and V. Faraoni, Rev. Mod. Phys. 82, 451 (2010).

[7] S. Nojiri and S. D. Odintsov, Phys. Rep. 505, 59 (2011). S. Nojiri, S. D. Odintsov, and V. K. Oikonomou, Phys. Rep. 692, 1 (2017).

[8] M. Tegmark et al., Astrophys. J. 606, 702 (2004).

[9] M. Kowalski et al., Astrophys. J. 686, 749 (2008).

[10] E. Komatsu et al., Astrophys. J. Suppl. Ser. 180, 330 (2009).

[11] P. A. R. Ade et al. (Planck Collaboration), Astron. Astrophys. 594, A13 (2016).

[12] S. Basilakos, S. Nesseris, and L. Perivolaropoulos, Phys. Rev. D 87, 123529 (2013).T. Liu, X. Zhang, andW. Zhao, Phys. Lett. B 777, 286 (2018).

[13] R. Ferraro and F. Fiorini, Phys. Rev. D 75, 084031 (2007).

[14] B. Li, J. D. Barrow, and D. F. Mota, Phys. Rev. D 76, 044027 (2007).

[15] S. Nojiri and S. D. Odintsov, Phys. Lett. B 631, 1 (2005).

[16] G. Cognola, E. Elizalde, S. Nojiri, S. D. Odintsov, and S. Zerbini, Phys. Rev. D 73, 084007 (2006).

[17] M. J. S. Houndjo, M. E. Rodrigues, N. S. Mazhari, D. Momeni, and R. Myrzakulov, Int. J. Mod. Phys. D 26, 1750024 (2017).

[18] A. De Felice, J.-M. Gerard, and T. Suyama, Phys. Rev. D 82, 063526 (2010).

[19] T. Harko, F. S. N. Lobo, S. Nojiri, and S. D. Odintsov, Phys. Rev. D 84, 024020 (2011).

[20] T. Clifton, P. G. Ferreira, A. Padila, and C. Skordis, Phys. Rep. 513, 1 (2012).

[21] T. Clifton, Classical Quantum Gravity 23, 7445 (2006).

[22] J. A. Leach, S. Carloni, and P. K. S. Dunsky, Classical Quantum Gravity 23, 4915 (2006).

[23] T. Clifton, Classical Quantum Gravity 24, 5073 (2007).

[24] T. Clifton and J. D. Barrow, Classical Quantum Gravity 23, 2951 (2006).
[25] A. Paliathanasis, Phys. Rev. D 95, 064062 (2017).

[26] S. Nesseris, S. Basilakos, E. N. Saridakis, and L. Perivolaropoulos, Phys. Rev. D 88, 103010 (2013).

[27] R. C. Nunes, A. Bonilla, S. Pan, and E. N. Saridakis, Eur. Phys. J. C 77, 230 (2017).

[28] R. C. Nunes, S. Pan, E. N. Saridakis, and E. M. C. Abreu, J. Cosmol. Astropart. Phys. 01 (2017) 005.

[29] C. Daskaloyannis and K. Ypsilantis, J. Math. Phys. (N.Y.) 47, 042904 (2006).

[30] S. Cotsakis, J. Demaret, Y. De Rop, and L. Querella, Phys. Rev. D 48, 4595 (1993).

[31] S. Cotsakis and P. G. L. Leach, J. Phys. A 27, 1625 (1994).

[32] C. Scheen and J. Demaret, Classical Quantum Gravity 13, 1909 (1996).

[33] J. Demaret and C. Scheen, J. Phys. A: Math. Gen. 29, 59 (1996).

[34] S. Cotsakis, G. Kolionis, and A. Tsokaros, Phys. Lett. B 721, 1 (2013).

[35] S. Cotsakis, S. Kadry, G. Kolionis, and A. Tsokaros, Phys. Lett. B 755, 387 (2016).

[36] A. Paliathanasis, J. D. Barrow, and P. G. L. Leach, Phys. Rev. D 94, 023525 (2016).

[37] A. Paliathanasis, Eur. Phys. J. C 77, 438 (2017).

[38] J. Latta, G. Leon, and A. Paliathanasis, J. Cosmol. Astropart. Phys. 11 (2016) 051.

[39] L. V. Ovsiannikov, Group Analysis of Differential Equations (Academic Press, New York, 1982).

[40] K. Rosquist and C. Uggla, J. Math. Phys. (N.Y.) 32, 3412 (1991).

[41] S. Capozziello, R. De Ritis, C. Rubano, and P. Scudellaro, Riv. Nuovo Cimento 19, 1 (1996).

[42] S. Cotsakis, P. G. L. Leach, and H. Pantazi, Gravitation Cosmol. 4, 314 (1998).

[43] B. Vakili, Phys. Lett. B 664, 16 (2008).

[44] S. Capozziello, E. Piedipalumbo, C. Rubano, and P. Scudellaro, Phys. Rev. D 80, 104030 (2009).

[45] Y. Zhang, Y.-G. Gong, and Z.-H. Zhu, Phys. Lett. B 688, 13 (2010).

[46] H. M. Sadjadi, Phys. Lett. B 718, 270 (2012). 
[47] B. Vakili and F. Khazaie, Classical Quantum Gravity 29, 035015 (2012).

[48] K. Atazadeh and F. Darabi, Eur. Phys. J. C 72, 2016 (2012).

[49] F. Darabi, K. Atazadeh, and A. Rezaei-Aghdam, Eur. Phys. J. C 73, 2657 (2013).

[50] H. Dong, J. Wang, and X. Meng, Eur. Phys. J. C 73, 2543 (2013).

[51] B. Vakili, Phys. Lett. B 738, 488 (2014).

[52] T. Christodoulakis, N. Dimakis, P. A. Terzis, and G. Doulis, Phys. Rev. D 90, 024052 (2014).

[53] P. A. Terzis, N. Dimakis, and T. Christodoulakis, Phys. Rev. D 90, 123543 (2014).

[54] N. Dimakis, T. Christodoulakis, and P. A. Terzis, J. Geom. Phys. 77, 97 (2014).

[55] N. Dimakis, A. Giacomini, S. Jamal, G. Leon, and A. Paliathanasis, Phys. Rev. D 95, 064031 (2017).

[56] F. Schwarz, J. Phys. A: Math. Gen. 16, L133 (1983).

[57] A. Paliathanasis, M. Tsamparlis, and S. Basilakos, Phys. Rev. D 90, 103524 (2014).

[58] G. Papagiannopoulos, S. Basilakos, J. D. Barrow, A. Giacomini, and A. Paliathanasis, Phys. Rev. D 95, 024021 (2017).

[59] A. Paliathanasis, M. Tsamparlis, and S. Basilakos, Phys. Rev. D 84, 123514 (2011).

[60] A. Paliathanasis, Classical Quantum Gravity 33, 075012 (2016).

[61] C. Gao, Y. Gong and X. Wang, and X. Chen, Phys. Lett. B 702, 107 (2011).

[62] J. Klusoň, Classical Quantum Gravity 28, 125025 (2011).

[63] A. Cid and P. Labrana, Phys. Lett. B 717, 10 (2012).
[64] J. O’Hanlon, Phys. Rev. Lett. 29, 137 (1972).

[65] T. P. Sotiriou, Gravity, and scalar fields, in Proceedings of the 7th Aegean Summer School: Beyond Einstein's theory of gravity, Modifications of Einstein's Theory of Gravity at Large Distances, Paros, Greece, edited by E. Papantonopoulos, Lect. Notes Phys. 892 (2015).

[66] R. Ferraro, AIP Conf. Proc. 1471, 103 (2012).

[67] V. I. Arnol'd, Mathematical Methods in Classical Mechanics, Graduate texts in Mathematics 60, 2nd ed (Springer, New York, 1991).

[68] A. A. Starobinsky and H. J. Schmidt, Classical Quantum Gravity 4, 695 (1987).

[69] J. D. Barrow, Phys. Rev. D 89, 064022 (2014).

[70] A. Paliathanasis, J. Cosmol. Astropart. Phys. 08 (2017) 027.

[71] R. M. Hawkins and J. E. Lidsey, Phys. Rev. D 66, 023523 (2002).

[72] F. L. Williams and P. G. Kevrekidis, Classical Quantum Gravity 20, L177 (2003).

[73] M. Tsamparlis and A. Paliathanasis, J. Phys. A 45, 275202 (2012).

[74] F. Canfora, A. Paliathanasis, T. Taves, and J. Zanelli, Phys. Rev. D 95, 065032 (2017).

[75] H. R. Lewis, Phys. Rev. Lett. 18, 510 (1967).

[76] T. Barreiro, E. J. Copeland, and N. J. Nunes, Phys. Rev. D 61, 127301 (2000).

[77] H. Motohashi and A. A. Starobinsky, Eur. Phys. J. C 77, 538 (2017).

[78] W. Sarlet and F. Cantrijin, SIAM Rev. 23, 467 (1981).

[79] M. Crampin, Rep. Math. Phys. 20, 31 (1984). 